\title{
Incidence of insulin dependent diabetes mellitus in Karachi, Pakistan
}

\author{
A Staines, S Hanif, S Ahmed, P A McKinney, S Shera, H J Bodansky
}

\begin{abstract}
Objectives-To determine the incidence of insulin dependent diabetes mellitus (IDDM) among children aged up to 16 years residing in the city of Karachi, Pakistan, during the five years from 1989 to 1993.

Design-Retrospective study of incidence using hospital and clinic records.

Setting-The city of Karachi, Pakistan.

Subjects-Children satisfying standard criteria for the diagnosis of IDDM, attending treatment facilities for the first time during the study period.

Main outcome measures-The incidence of IDDM in this population and its variation by age and sex.

Results-The incidence of IDDM in this population is $1.02 / 100000$ per year, which is one of the lowest incidence rates yet reported.

Conclusions-The very low incidence of IDDM, contrasted with the substantially higher incidence among migrants, supports the view that environmental factors are the major determinants of variations in the incidence of this condition between populations.

(Arch Dis Child 1997;76:121-123)
\end{abstract}

Keywords: insulin dependent diabetes mellitus; disease incidence; Pakistan.

The incidence of childhood insulin dependent diabetes mellitus (IDDM) is known to vary widely between countries. Rates among children aged 16 years and under vary from over 40/100 000 in Finland to less than 1/100 000 in Japan. ${ }^{12}$ In most European registries the rate is between 5 and 20/100 000 per year, with the higher rates generally in northern Europe and lower rates in southern Europe, apart from Sardinia. ${ }^{1}$ The reasons for this variation are unclear and the relative contributions of genetics and environment are a subject of debate.

The incidence of IDDM in India and Pakistan is not known, but it is believed to be low. This is suggested by several prevalence studies from India and Pakistan..$^{3-5}$ No study of the incidence of IDDM has yet been published from any part of the Indian subcontinent, however.

The city of Bradford in West Yorkshire has a large population of children whose parents were migrants from rural Pakistan in the 1950s and 1960s. Children with IDDM from this group have the same high risk HLA markers and the same autoantibodies as other local children with IDDM. ${ }^{6}$ These children of migrants had a very low incidence of IDDM in the late 1970s. This has risen steadily since then and is now only slightly lower than that found in other local children. ${ }^{78}$

To explore these results further and to obtain the first set of incidence data from the Indian subcontinent, we carried out a study of the incidence of IDDM in the city of Karachi, Pakistan. Karachi is a large city, and a former capital, on the coast of Pakistan. The population is almost entirely Moslem. It is an important industrial centre and a major port.

\section{Methods}

All of the facilities in Karachi treating children with IDDM were identified by questioning local doctors. All of the larger facilities agreed to cooperate with this study; however, two small private hospitals declined to take part. Each identified centre was visited. Cases were identified using clinic and ward records and hospital notes were abstracted onto a standard form.

Children aged 16 years or under at diagnosis, diagnosed between 1989 and 1993 and normally resident in the city of Karachi at the time of diagnosis, were eligible for inclusion. Routine demographic information was recorded for each child, including dates of birth and diagnosis, sex, home address, and family history of diabetes.

It was not possible to apply capturerecapture methods to estimate our completeness of ascertainment as no independent secondary source of cases could be found. ${ }^{9}$ Pharmacy records for insulin prescriptions were not available, either centrally or in the pharmacies themselves. The records of the Diabetes Association of Pakistan were investigated, but their facilities provide treatment for members and, although it was an important primary source for cases, there was negligible overlap with other clinics. Primary care records are not centralised and patients often consult several different doctors and a range of traditional healers. No central records of hospital admissions exist.

The most recent census of Pakistan was in 1981 and this serves as the basis for population estimates. The census bureau made available to us local estimates for the population of Karachi by sex and five year age groups for each year from 1981 to 1995 (Pakistan Bureau of the Census, personal communication). These were derived by the Census Bureau from a simple growth model. Calculations were also per- 
Table 1 Rates (number of cases) of IDDM/100 000 children/year in Karachi from 1989 to 1993 inclusive

\begin{tabular}{llll}
\hline Age (years) & Boys & Girls & Total \\
\hline $0-4$ & $0.7(23)$ & $0.5(15)$ & $0.6(38)$ \\
$5-9$ & $0.9(31)$ & $0.6(18)$ & $0.8(49)$ \\
$10-14$ & $1.3(41)$ & $2.1(57)$ & $1.7(98)$ \\
$15-16$ & $1.1(12)$ & $1.2(11)$ & $1.2(23)$ \\
Total & $1.01(107)$ & $1.04(101)$ & $1.02(208)$ \\
\hline
\end{tabular}

formed using the 1981 census as a base to give a partial check on the impact of errors in the population estimates on our results.

\section{Results}

The 1981 census recorded 2.5 million children aged 16 years and under in Karachi, 1.2 million girls and 1.3 million boys. The estimated 1991 population was 4.1 million children, 2 million girls and 2.1 million boys.

Two hundred and forty cases were identified aged 16 years or under and diagnosed between 1989 and 1993. Two hundred and eight were eligible as they were resident in Karachi at the time of diagnosis. The other 32 children had moved to Karachi for treatment after diagnosis elsewhere. There were 107 boys and 101 girls. The incidence per 100000 per year was 1.01 for boys, 1.04 for girls and 1.02 in total, one of the lowest figures yet recorded (table 1 ). If the 1981 population is taken as a base the total rate is $1.67 / 100000$ per year.

The peak incidence was between the ages of 10 and 12 years in both sexes, which is similar to the pattern usually seen in registries from Western Europe ${ }^{10}$ (fig 1). The highest rate was for girls aged 12 years. There was a slight female excess, rather than the male excess found in most registries, but this was not statistically significant (rate ratio $1.03 ; 95 \%$ confidence interval 0.78 to 1.36 ).

\section{Discussion}

Diabetes is one of the most important chronic diseases of childhood. ${ }^{11} \mathrm{~A}$ better understanding of its aetiology might prove beneficial to public health. Knowledge of the incidence of IDDM among children in different parts of the world is an important tool for epidemiology. Patterns of international variation in the incidence of IDDM provide material with which to develop

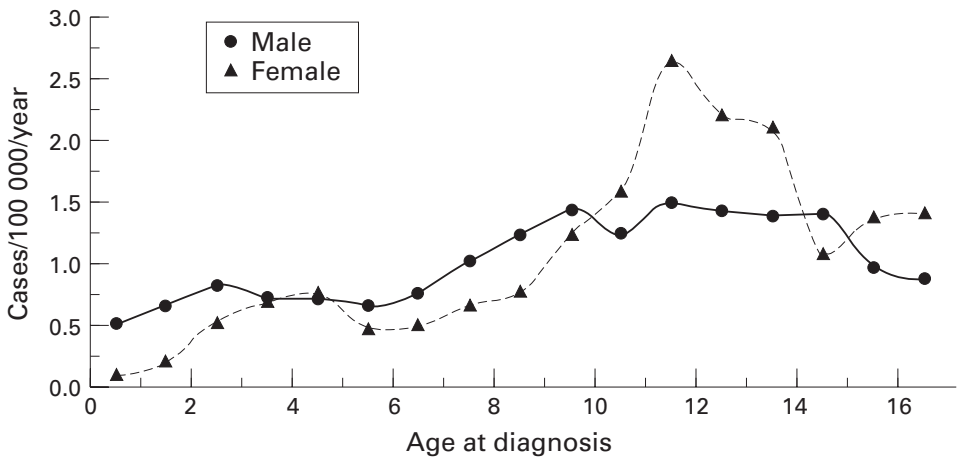

Figure 1 Incidence of IDDM/100 000 children/year in Karachi from 1989 to 1993 plotted against age and smoothed with a three year moving average. and test hypotheses about the origins of this serious disease. This is the first such report from the Indian subcontinent.

This study confirms the exceptionally low incidence of IDDM in Pakistan and validates our earlier study of migrants from Pakistan. ${ }^{7}$ Western Europeans and inhabitants of the northern part of the Indian subcontinent share common ancestry and close genetic and linguistic links, ${ }^{12}$ so these observations suggest the importance of environmental factors in the origins of IDDM. There are many important environmental differences between the UK and Pakistan, including differences of diet, wealth, climate, and child health. Which of these contribute to the observed difference in rates is not known.

Although our study certainly missed some cases it is likely that most of the incident cases have been recorded. Although it was not possible to confirm the completeness of our case ascertainment directly, our searches of the available records were extensive and thorough. It is unlikely that a large proportion of cases reaching medical treatment centres could have been missed. It is the opinion of doctors in Karachi that almost all cases do reach medical care. Only $6 \%$ of the children in this study had attended traditional healers for their disorder (S Hanif and S Ahmed, unpublished observations). Furthermore, even an ascertainment rate as low as $50 \%$, far worse than is likely, would not modify our conclusions significantly. The population estimates are also of unknown reliability. They are derived for use by planners and others working in the city, and are the best figures available. Even large errors in population estimates would not affect our conclusions.

This study shows the value of careful comparative studies of disease incidence between countries. Establishing that a difference exists between two countries and that it disappears in migrants from one country to the other is only a beginning. Understanding the reasons for occurrence is a possible route to a better understanding of the origins of IDDM and further work to this end is planned.

1 Green A, Gale EA, Patterson CC. Incidence of childhoodonset insulin-dependent diabetes mellitus: the EURODIAB ACE Study. Lancet 1992;339:905-9.

2 Matsuura N, Fukushima N, Fujita $\mathrm{H}$, et al. Epidemiologic survey of juvenile-onset insulin dependent diabetes mellisurvey of juvenile-onset insulin dependent diabetes melltus (IDDM) in Hokkaido, Japan

3 Krishnaswami CV, Chandra P. The significance of certain epidemiological variants in the genesis of juvenile insulindependent diabetes mellitus-the need for a global program of co-operation. Tohoku $\mathcal{f}$ Exp Med 1983; 141 (suppl): $161-70$

4 Menon PN, Viramani A, Shah P, Raju R, Sethi AK. Childhood onset diabetes mellitus in India: an overview. International fournal of Diabetes in Developing Countries 1990;10:11-16.

5 Ramachandran A, Snehalatha C, Abdul-Khader OMS, Joseph TA, Viswanathan M. Prevalence of childhood diabetes in an urban population in South India. Diabetes Res Clin Pract 1992;17:227-31.

6 Bodansky HJ, Beverley DW, Gelsthorpe K, Saunders A, Bottazzo GF, Haigh D. Insulin dependent diabetes in Bottazzo GF, Haigh D. Insulin depe
Asians. Arch Dis Child 1987;62:227-30.

7 Bodansky HJ, Staines A, Stephenson C, Haigh D, Cartwright R. Evidence for an environmental effect in the aetiology of insulin-dependent diabetes mellitus in a transaetiology of insulin-dependent diabetes mellitus
migratory population. BMF 1992;304:1020-2. 
8 Staines A, Bodansky HJ, Lilley HEB, Stephenson C, McNally RJQ, Cartwright RA. The epidemiology of childhood diabetes mellitus in the United Kingdom: the Yorkshire Regional Childhood Diabetes Register. Diabetologia 1993;36:1282-7.

9 Hook EB, Regal RR. Validity of Bernouilli census, log-linear and truncated binomial models for correcting for underascertainment in prevalence studies. Am f Epidemiol 1988; 116:168-176.
10 Levy-Marchal C, Patterson CC, Green A. Variation by age group and seasonality at diagnosis of childhood IDDM in Europe. Diabetologia 1995;38:823-30.

11 Gortmaker SL, Sappenfield W. Chronic childhood disorders: prevalence and impact. In: Haggerty RJ, ed. Symposium on chronic disease in children. Pediatr Clin North Am 1984;31:3-18

12 Cavalli-Sforza LL, Menozi P, Piazza A. The history and geography of human genes. Princeton: Princeton University Press, 1994.

\section{Shaken and stirred}

Whiplash shaken infant syndrome (WSIS) has two main causes, motor accidents and child abuse. The acute syndrome is well described and short term follow up studies have suggested that up to half of the children have fully recovered after a year. A recent report from Brussels, however, has shown that there is often a latent period after initial recovery and before the long term sequelae become evident (Christine Bonnier and colleagues, Developmental Medicine and Child Neurology 1995;37: 943-56).

Thirteen children with severe WSIS due to child abuse were admitted over eight years to a hospital with a multidisciplinary child abuse service and followed up for up to 14 years. There was one early death and at necopsy that infant had widespread intracerebral and pericerebral blood vessel damage, section of the cerebral peduncles, and tears due to shearing between cerebral hemisphere grey and white matter.

Of the 12 survivors, six were severely affected from the start and did badly but the other six initially recovered and were considered normal two months after the incident. Only one of these remained normal on follow up to aged 5. Five showed further neurological disability after latent intervals of between six months and five years. All five had global learning disorders with measured IQ of between 53 and 80. Two developed a hemiparesis, three had behavioural problems, and all needed special education. The deficits appeared in chronological sequence: slowing of brain growth (four months after injury), long tract signs (six to 12 months), epilepsy (up to two years), and behavioural and psychological problems (three to six years).

Severely shaken children need prolonged follow up and early return to normality should not be taken as an indication that all is well.

ARCHIVIST 